Provided for non-commercial research and educational use only. Not for reproduction or distribution or commercial use.

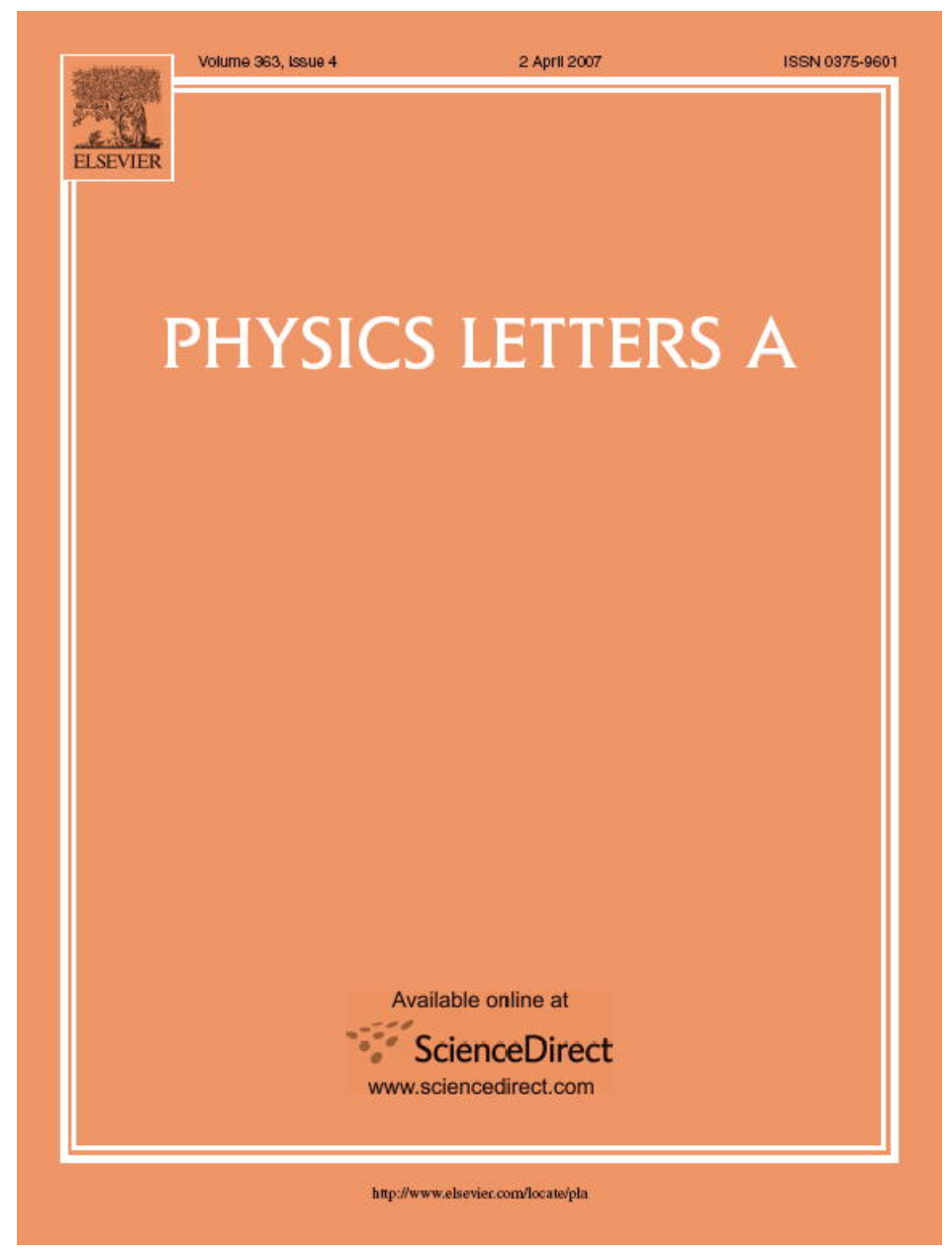

This article was originally published in a journal published by Elsevier, and the attached copy is provided by Elsevier for the author's benefit and for the benefit of the author's institution, for non-commercial research and educational use including without limitation use in instruction at your institution, sending it to specific colleagues that you know, and providing a copy to your institution's administrator.

All other uses, reproduction and distribution, including without limitation commercial reprints, selling or licensing copies or access,

or posting on open internet sites, your personal or institution's website or repository, are prohibited. For exceptions, permission may be sought for such use through Elsevier's permissions site at: 


\title{
Characterizing correlation changes of complex pattern transitions: The case of epileptic activity
}

\author{
Gerold Baier ${ }^{\mathrm{a}, *, 1}$, Markus Müller ${ }^{\mathrm{a}}$, Ulrich Stephani ${ }^{\mathrm{b}}$, Hiltrud Muhle ${ }^{\mathrm{b}}$

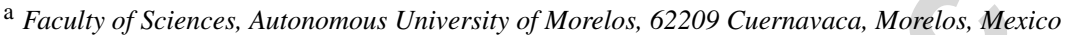 \\ b Clinic for Neuropediatrics, University of Kiel, 24105 Kiel, Germany \\ Received 29 November 2005; accepted 1 November 2006 \\ Available online 29 November 2006 \\ Communicated by A.P. Fordy
}

\begin{abstract}
We analyze the time-dependent spectrum of eigenvalues of the correlation matrix for multivariate EEG data at the transition to epileptic seizures. By a mechanism of level repulsion between states at both edges of the spectrum of the correlation matrix, relevant information about quantitative correlation changes is reflected in the largest and smallest eigenvalues and corresponding eigenvectors. By the application of measures from random matrix theory we provide evidence that statistically relevant information can be obtained both at the upper and the lower end of the spectrum. In addition, information about spatial characteristics of correlation changes can be extracted.
\end{abstract}

(c) 2006 Elsevier B.V. All rights reserved.

PACS: 02.50.Sk; 05.45.Tp; 89.75.Fb

Keywords: Time series analysis; Correlation matrix; Random matrix theory; Pattern transitions; Epilepsy

\section{Introduction}

The spectrum of eigenvalues of the equal-time correlation matrix $\mathbf{C}$ offers information about correlations in multivariate data sets with highly complex, noise-like behavior (see e.g. [13]). The foundation for this are results from random matrix theory about the spectra of matrices composed from uncorrelated time series (forming the so-called Wishart ensemble, WE) [4]. A number of methods have been devised to detect deviations from this extreme case of matrix entries formed by uncorrelated data. These methods have been applied successfully to the analysis of data sets like multivariate time series from stock markets (e.g. see [2,3]). A common starting point for these methods is the evaluation of the distribution of eigenvalues in the case of correlation matrices with a large number of time series. If some eigenvalues are found to separate from

\footnotetext{
* Corresponding author.

E-mail address: baier@buzon.uaem.mx (G. Baier).

1 Present address: EML Research, Schloss-Wolfsbrunnenweg 33, 69118 Heidelberg, Germany.
}

the "bulk" of eigenvalues (which is supposed to be described by the distribution of the Wishart ensemble), they are interpreted as containing information about (non-random) correlations. For stock market data with time series available for some hundreds of stocks, this yielded a small number of eigenvalues deviating significantly from the range predicted by the Wishart distribution, mainly at the upper edge of the spectrum [2,3]. In [5] artificial data were analyzed, that showed how both the upper and the lower edge of the spectrum of eigenvalues contain information about non-random correlations. Here we apply the concepts to data from human electroencephalogram (EEG) and show how useful information can be extracted.

Electric activity of the human brain as picked up by the EEG with scalp electrodes displays irregular spatio-temporal patterns with comparatively weak correlations between the time series during periods of conscious rest. Epileptic activity occurs in the form of hypersynchronized electric neural activity expanded over large regions of the cortex resulting in a notable reduction of the effective number of degrees of freedom of the brain dynamics. In the case of so-called absence seizures this reduction is supposed to occur abruptly and is visible in the record- 
ings of most or all surface electrodes simultaneously. In the EEG measurements this leads to patterns with characteristically altered auto- and cross-correlations. Auto-correlation changes can readily be quantified with the autocorrelation function or the Fourier spectrum for each time series. Cross-correlations are less handy because there are $(M-1) M / 2$ cross-correlation coefficients, where $M$ is the number of time series. Thus, tools are required that allow the analysis of both short- and long-range correlation changes in spatio-temporal systems.

For the case of standard EEG with 19 scalp electrodes, only small ensembles of correlation matrices can be created over comparatively short time segments due to the non-stationarity of the data set. It is then not reasonable to try to estimate a continuous distribution function from the 19 eigenvalues (e.g. in order to compare it with the Wishart ensemble). However, one may take advantage of the fact that the dynamics of the comparatively small number of eigenvalues can be studied individually. For artificially generated time series it was shown that a small number of time series allows a differentiation of the scales on which cross-correlations occur [5]. It was emphasized that details about the correlation changes can be extracted if both the largest and the smallest eigenvalues of the correlation matrix are considered. If the time series are uncorrelated the eigenvalues are randomly distributed around 1. Correlations then lead to level repulsion between the largest and smallest eigenvalues. More specifically, if $k$ time series are correlated, $\mathrm{k}$ eigenvalues at both ends of the spectrum repel. Furthermore, in some cases, especially for subtle changes of the correlation structure within a subset of the multivariate data, the information obtained from the lower edge of the spectrum might be more significant [5]. Here, we show how the spectrum of eigenvalues allows to study complex correlation changes in experimental data sets.

\section{Method and data}

For the analysis of the temporal evolution of two-point correlations in a data set a running window of $\mathbf{C}$ is defined. For a time window $\Delta t$ containing $N$ data points, $\mathbf{C}$ is obtained as:

$\mathbf{C}\left(t_{l}\right)=\frac{1}{N} \sum_{k=1}^{N} \tilde{X}_{i}\left(t_{k}\right) \tilde{X}_{j}\left(t_{k}\right)$,

where $i, j=1, \ldots, M, M$ is the number of time series $(M=19$ for standard EEG), and $t_{l}$ is the central value of actual time window $\Delta t$. The normalized time series are given by

$\tilde{X}_{i}=\left(X_{i}\left(t_{k}\right)-\bar{X}_{i}\right) / \sigma_{i}$,

where $\bar{X}_{i}$ is the mean and $\sigma_{i}$ the standard deviation of $X_{i}$ calculated over the actual time window of length $\Delta t$.

This segment is shifted with maximal overlap over the time series. The value of $\Delta t$ has to be selected in accordance with the temporal resolution of the measurement and the problem to be studied. It is chosen such that it allows high temporal resolution without obscuring changes by noise level and random correlations. Furthermore, the choice of $\Delta t$ defines the time scale within which correlations can be observed. In order to observe dynamic changes that are presumably in the range of seconds for absence seizures [6], we varied $\Delta t$ between 0.3 seconds and 4 seconds and found 1.0 seconds (corresponding to 256 data points) to be optimal in the sense that it reduces noise-induced fluctuations while maintaining a good temporal resolution.

For a detailed interpretation of the temporal evolution of correlation changes in a multivariate data set, a method is needed which (a) reduces the number of analyzed quantities (in our case $M(M-1) / 2$ cross-correlation coefficients) without loosing spatial information about the correlation structure; and (b) separates random correlations and noise from genuine information. This can be done by the evaluation of the eigenvalues (and eigenvectors) of the correlation matrix $\mathbf{C}$ [5].

Clinical EEGs were recorded with standard 10/20 position of 19 scalp electrodes (sampling rate: 256 data points per second) from patients (age 8-13, two male, three female) with diagnosed primary generalized absence seizures of $3 \mathrm{~Hz}$ spikeand-wave appearance. High-pass $(>0.5 \mathrm{~Hz})$ filtered samples of several minutes length were selected. These included at least one spontaneous transition from apparently normal to seizure activity in the awake state. Data were transformed according to the Hjorth source derivation [7] and filtered for $50 \mathrm{~Hz}$ AC contamination. The signals were normalized (average 0 , amplitude variance 1 according to Eq. (2)).

\section{Results}

We performed the analysis of the time-dependent eigenvalues of $\mathbf{C}$ for 5 patients with a total of 9 spontaneous seizures. For the analysis of a multivariate dynamics of cross-correlations we calculated the time-dependent eigenvalues of $\mathbf{C}$. Fig. 1a shows an exemplary time series of one of the electrodes in patient 1 . The chosen period of the time series includes seizurefree and seizure activity (the latter easily recognizable by the increased amplitude of the spike-wave complexes at about 443 to 452 seconds). The largest and smallest eigenvalues in Figs. 1b and 1c show noisy fluctuations around a comparatively constant mean prior to the seizure and after the seizure. Of the four largest eigenvalues displayed in Fig. 1b, $\lambda_{19}$ appears to be set apart from the triplet $\lambda_{18}$ through $\lambda_{16}$ before and after the seizure. During the seizure, the mean value of $\lambda_{19}$ is slightly increased but due to the strong fluctuations before and after the seizure this increase is not significant. Eigenvalue $\lambda_{18}$, however, rises significantly to a higher level during the seizure and remains separated from its two smaller neighbors until the seizure terminates. Such a behavior can be interpreted as the formation of two essentially uncorrelated clusters of time series [5]. In addition, due to decreased fluctuations in all eigenvalues during the seizure, the separation of the two largest eigenvalues becomes even more clearly pronounced. This is evidence of a period of a global increase in correlations.

Further information is obtained from the inspection of the four smallest eigenvalues (Fig. 1c). The smallest eigenvalue $\lambda_{1}$ is separated from its larger neighbors in the non-epileptic parts of the time series. This is probably due to the presence of static correlations, e.g. from the choice of reference. The other three eigenvalues stay close to each other and close to the group formed by eigenvalues $\lambda_{5}$ through $\lambda_{18}$. The fluctuations of the 

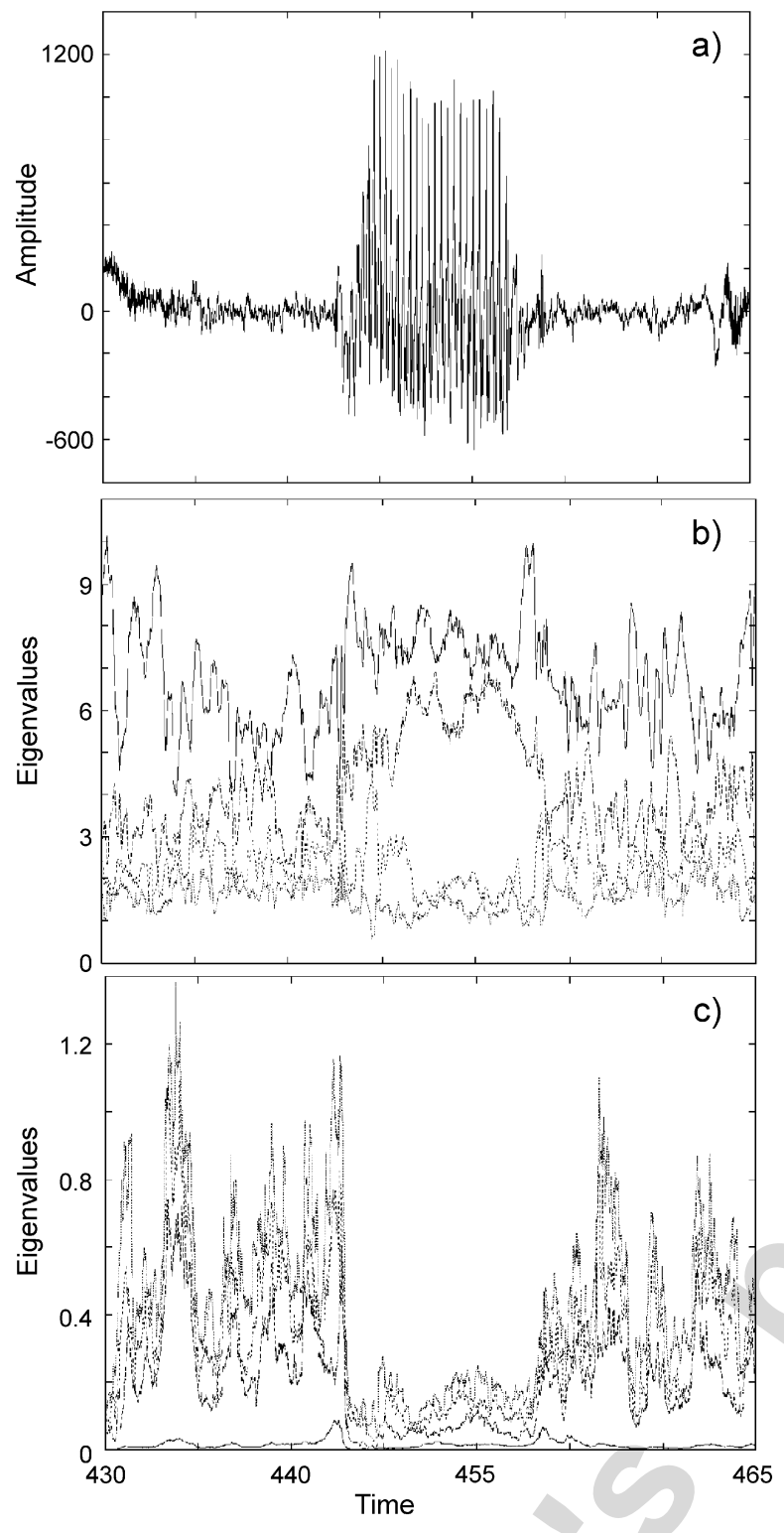

Fig. 1. (a) Time series of electrode $\mathrm{C} 3$ before, during, and after absence seizure 1 in patient 1. (b) The four largest eigenvalues of $\mathbf{C}$ (from top to bottom) for the complete EEG during the same period. (c) The four smallest eigenvalues of $\mathbf{C}$ (from top to bottom). For panels (b) and (c) is $\Delta t=1 \mathrm{~s}$.

small eigenvalues except $\lambda_{1}$ are large during the non-epileptic phases. During the epileptic phase eigenvalues $\lambda_{2}$ through $\lambda_{16}$ decrease in mean value and variance (shown exemplarily by the decrease of $\lambda_{2}$ through $\lambda_{4}$ in Fig. 1c) and together with $\lambda_{1}$ stay at a low level until the seizure terminates and the pre-seizure pattern is restored qualitatively.

Analyzing seizures from different patients we found that the cross-correlation pattern of the EEG during seizure-free activity is comparatively stable and that it deviates significantly from the pattern during the seizure. Thus, within the resolution given by the choice of $\Delta t$, the spectrum of eigenvalues shows both the nontrivial background correlation structure and the temporal correlation changes in the EEG originating from the epileptic seizure. We find that for other seizures of the same type the behavior of the eigenvalues is qualitatively as in Figs. 1b and 1c.

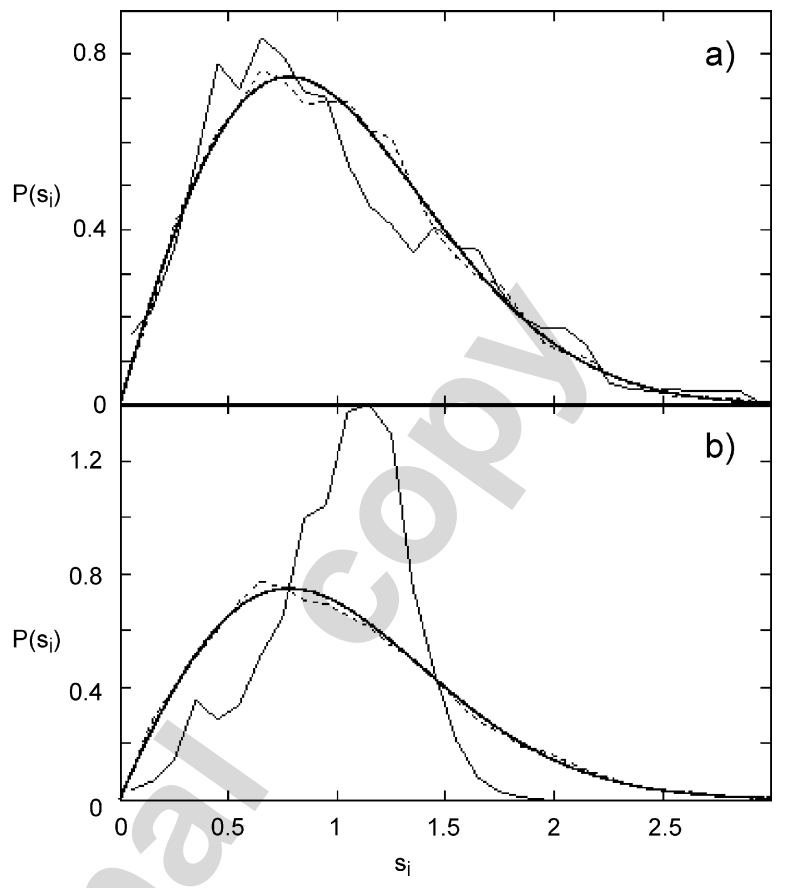

Fig. 2. Normalized $P(s)$ distribution of differences $S_{18}$ (a) and $S_{17}$ (b) in 2 min interval prior the seizure (dashed line) and in the 12 seconds seizure interval (thin solid line) in seizure 1 of patient $1 . \Delta t=0.35 \mathrm{~s}$. For comparison, the theoretical curve for the GOE (not the Wigner surmise) is drawn with a thick solid line.

Only, seizure 3 of patient 5 presents a more notable exception from the correlation pattern described so far. Here, only the largest eigenvalue $\lambda_{19}$ increases significantly during the seizure while $\lambda_{18}$ maintains its pre-seizure mean.

For further analysis of the correlation behavior we use measures derived from random matrix theory (RMT) [8]. We are interested in the relative changes of the eigenvalues, we chose to evaluate differences between levels. We first identify intervals of interest and for each window we construct an ensemble of matrices with $\Delta t=90$ data points (an interval that is of the length of one complete spike-wave complex). For the ensemble of matrices in the chosen intervals we calculate distributions of the $M-1$ normalized differences between neighbors of the spectrum (in increasing order) for each interval [9]:

$S_{i}=\left(\lambda_{i+1}-\lambda_{i}\right) /\left\langle\lambda_{i+1}-\lambda_{i}\right\rangle$,

where $\langle\cdots\rangle$ denotes the average over the ensemble.

Fig. 2 shows two of the resulting $P(s)$ distributions in a 2 min interval prior the seizure, which serves as our reference interval with normal background activity (yielding 30630 matrices), and in the 12 seconds seizure interval (yielding 2982 matrices). The experimentally obtained results are drawn in comparison with the theoretically known distribution for the Gaussian Orthogonal Ensemble (GOE) which is supposed to be the null hypothesis of the uncorrelated state. The nearest neighbor distributions $S_{18}$ (Fig. 2a) are similar in the two chosen intervals and both lie close to the GOE. Calculating the Kolmogorov-Smirnow similarity to compare these two distributions yields a value close enough to 1 to be consistent with the null hypothesis that both distributions may originate from 
the same generating mechanism. For the nearest neighbor distributions of $S_{17}$ in the two intervals (Fig. 2b) the KS test yields a value close to zero, rejecting the null hypothesis. In the reference interval, the nearest neighbor distributions of $S_{17}$ indicates only random repulsions between the two respective eigenstates (induced by incidental correlations only), whereas the distribution $P\left(S_{17}\right)$ obtained from the seizure interval indicates a higher stiffness and hence strong genuine correlations.

If in a set of time series the correlations fluctuate strongly, the eigenvectors rotate strongly and consequently the norm of their means (calculated over a certain interval) tends to vanish. If however, the correlations are stationary in a given segment, the vectors display preferred orientations [5] and their norms converge to some finite value above zero. Therefore, we calcu-

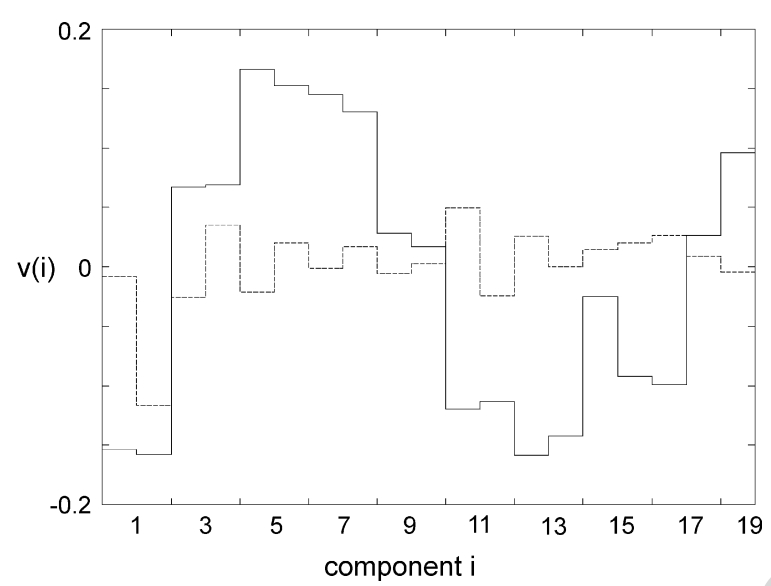

Fig. 3. Eigenvector components corresponding to the largest eigenvalue in the intervals of Fig. 2. Plotted are the mean values in $2 \mathrm{~min}$ interval prior the seizure (dashed line) and in the 12 seconds seizure interval (solid line) in seizure 1 of patient $1 . \Delta t=0.35 \mathrm{~s}$ late the mean eigenvectors corresponding to the 19 eigenvalues. Fig. 3 shows the 19 components of the mean eigenvector corresponding to the largest eigenvalue in the two minute pre-seizure interval and in the seizure interval (intervals as for Fig. 2). In the pre-seizure interval all components are comparatively small indicating strong variation in the correlation pattern (cf. [5]). In contrast, in the seizure interval most components have large absolute values, indicating a preferred orientation of this vector in the configuration space. In agreement with this finding are the values for the norm of the largest vector in the two intervals (as a measure of the preferred orientation or the stationarity of the data) which is 0.03 for the pre-seizure period and 0.11 for the seizure period. Only during the seizure interval the eigenvector corresponding to the largest eigenvalue shows a preferred orientation. The normal activity in the EEG displays a mostly random dynamics.

Next we investigate the transition from normal to seizure activity in more detail. In particular, we want to know how the changes from background activity to the electroencephalographically defined seizure activity occur and whether there are common features in different patients. To be able to tell whether a given observation is indeed related to the imminent epileptic dynamics or whether it is just incidental, quantities are required that allow the comparison between different instances of seizures. To be independent of absolute numerical values, we use the pre-seizure correlation pattern as a reference point to calculate the momentary eigenvalues relative to their pre-seizure mean as:

$\bar{\lambda}_{i}^{\mathrm{rel}}=\left(\lambda_{i}-\bar{\lambda}_{i}\right) / \bar{\lambda}_{i}$,

where the means of the $\bar{\lambda}_{i}$ are evaluated over a period of 2 minutes prior to the seizure. Fig. 4 shows the two largest and
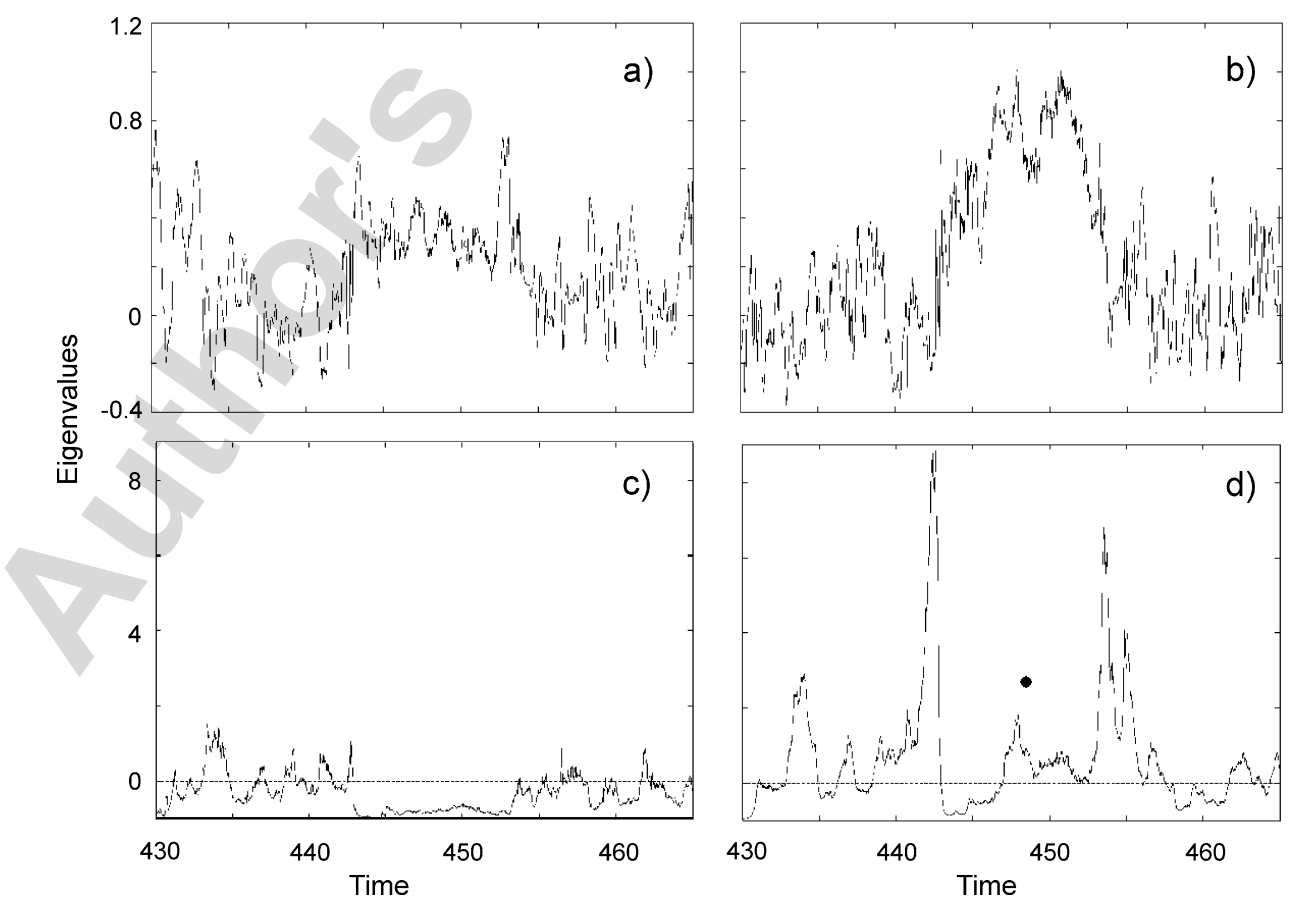

Fig. 4. Relative eigenvalues of $\mathbf{C}$ according to Eq. (3) for the same data as in Fig. 1 . Relative eigenvalue (a) $\lambda_{19}^{\text {rel }}$; (b) $\lambda_{18}^{\text {rel }}$; (c) $\lambda_{2}^{\text {rel }}$; and (d) $\lambda_{1}^{\text {rel }}$. The seizure occurs between 443 and 453 seconds. $\Delta t=1 \mathrm{~s}$. 
Table 1

Period of increased $\ddot{\lambda}_{1}^{\text {rel }}$ and maximum value of $\ddot{\lambda}_{1}^{\text {rel }}$ for absence seizures in different patients

\begin{tabular}{lcc}
\hline Patient & Duration (s) & Maximum \\
\hline 1, seizure 1 & 1.0 & 8.8 \\
1, seizure 2 & 6.0 & 15.9 \\
2, seizure 1 & 3.6 & 6.2 \\
2, seizure 2 & 3.5 & 5.0 \\
3, seizure 1 & 4.2 & 8.4 \\
4, seizure 1 & 2.3 & 5.2 \\
5, seizure 1 & 6.1 & 14.3 \\
5, seizure 2 & 5.1 & 5.9 \\
5, seizure 3 & 16.0 & 5.7 \\
\hline
\end{tabular}

a Due to artifacts average was taken over 70 seconds prior to seizure.

b Due to a previous epileptic activity the pre-seizure average $\bar{\lambda}_{1}$ could be evaluated only over 45 seconds.

two smallest relative eigenvalues of the period analyzed in Fig. 1.

The largest relative eigenvalue $\lambda_{19}^{\text {rel }}$ of seizure 1 in patient 1 (Fig. 4a) is elevated during the seizure to a value of approx. 0.3 , and the seizure period is clearly marked by the decrease of its variance. During the seizure, the second largest relative eigenvalue $\lambda_{18}^{\text {rel }}$ (Fig. 4b) reaches a mean of approximately 0.75 . In this case, the average increase is more significant than that for eigenvalue $\lambda_{19}^{\text {rel }} . \lambda_{18}^{\text {rel }}$ thereby undergoes the strongest change of all relative eigenvalues during the seizure as compared to the pre-seizure period. This confirms the strong separation of two largest eigenvalues during the seizure (cf. Figs. $1 \mathrm{~b}$ and $2 \mathrm{~b}$ ). The second smallest relative eigenvalue (Fig. 4c) decreases to a value of approximately -0.8 during the seizure and shows decreased fluctuations in this period (similar to the large relative eigenvalues). Qualitatively, the same as for $\lambda_{2}^{\text {rel }}$ can be said for relative eigenvalues $\lambda_{3}^{\text {rel }}$ through $\lambda_{16}^{\text {rel }}$. Only $\lambda_{17}^{\text {rel does not show }}$ significant changes during the seizure.

During the seizure, the smallest relative eigenvalue $\lambda_{1}^{\text {rel }}$ (Fig. 4d) first falls below, then rises above zero, but does not leave the range of fluctuations of the pre-seizure period. However, $\lambda_{1}^{\text {rel }}$ increases dramatically during an interval of about 2 seconds just before the clinical onset of seizure activity. Its value rises to a maximum of approximately 8.9 and then falls steeply at the onset of the seizure (at about 443 seconds). At the maximum, the corresponding ("absolute") eigenvalue $\lambda_{1}$ is about one order of magnitude larger than during the 2-minutes pre-seizure interval. When the seizure terminates, there is another increase in $\lambda_{1}^{\text {rel }}$ creating two maxima, after which the pre-seizure mean is approached again. The fact that only one eigenvalue rises indicates that only a small group of electrodes is involved and that the change leads to an overall correlation loss in the data set [5].

Repeating the analysis just described for another attack of the same patient and for attacks in other patients we find that for the behavior prior to the seizure a qualitatively similar picture of changes in the spectrum of eigenvalues as in the case presented was found for all seizures investigated ( 9 spontaneous absence seizures in 5 patients). Table 1 shows the periods for which $\lambda_{1}^{\text {rel }}$ stays outside the variance of the pre-seizure mean value and the maximal values of $\lambda_{1}^{\text {rel }}$ during this period. A significant increase

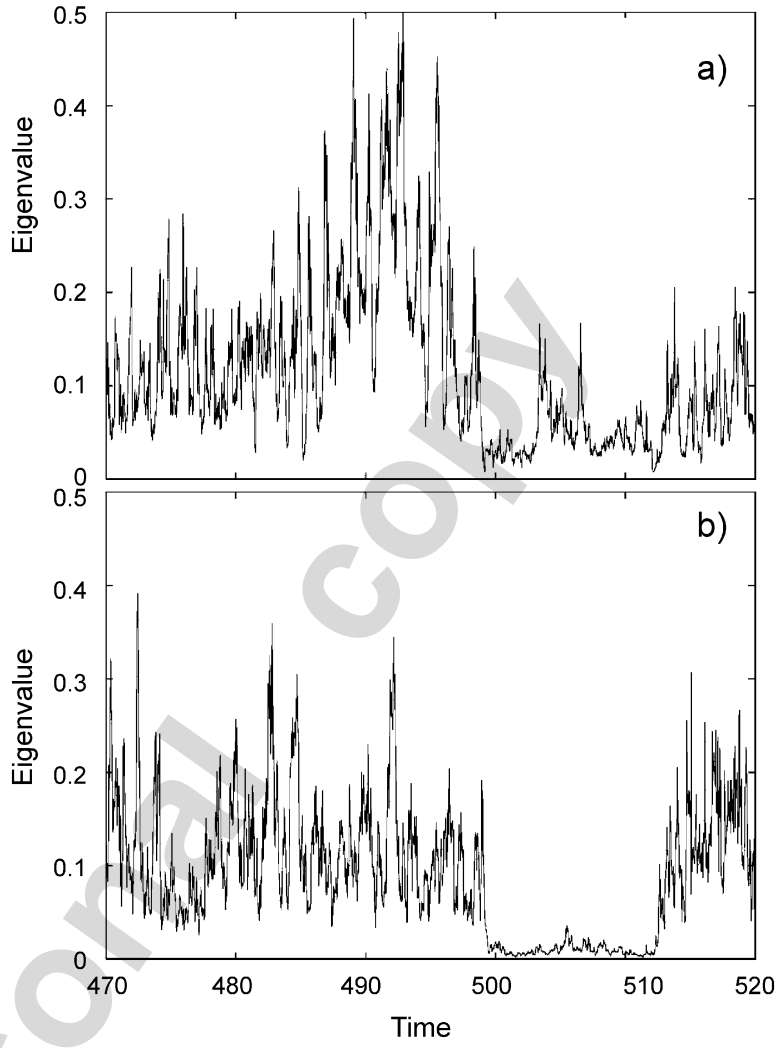

Fig. 5. Smallest eigenvalues for two subgroups of electrodes for seizure 3 in patient 5. (a) $\lambda_{1}$ of the group Fp1, Fp2, F4, F8, T4, Fz; (b) $\lambda_{1}$ of the group P3, P4, T5, T6, Cz, Pz. The seizure occurs from 498 to 512 seconds. $\Delta t=0.5 \mathrm{~s}$.

of $\lambda_{1}^{\text {rel }}$ is found in all cases. The periods for which this occurs vary from 1 to 15 seconds. In patient 5 a significant increase is found for more than one smallest eigenvalue prior to attacks. In seizure 3 of patient 5 the period of increased $\lambda_{1}^{\text {rel }}$ is the longest of all patients studied.

Applying RMT measures to the analysis of the smallest eigenvalues to a two minutes reference interval with background activity and a period of only 5 seconds prior to the seizure, we find that the distributions (as expressed by $P(s)$ ) for the difference between the two smallest eigenvalues shows a significant change. In the case of seizures 1 and 2 of patient 1 the KS similarity indices are much smaller than one, rejecting the null hypothesis that both distributions are from the same generating mechanism. In the case of seizure 3 from patient 5 we chose a 15 seconds interval to compare with the 2 minutes pre-seizure interval and obtain a KS value close to zero, again rejecting the null hypothesis. Similar to the case of artificial data (see [5]) we find that this result for the small eigenvalues is statistically more significant than that obtained for the large eigenvalues.

The inspection of the pattern of eigenvalues of patient 1 before the seizure indicates that the increase in $\lambda_{1}^{\text {rel }}$ may be related to cross-correlation changes that are confined to only a subgroup of the available time series [5]. This could be verified explicitly in seizure 3 of patient 5 which shows the most pronounced pre-seizure changes in both duration and the number of small eigenvalues affected. Sets of 6 time series were 
grouped together and their spectrum of 6 eigenvalues calculated in a time-dependent fashion as before. Fig. 5 shows the corresponding smallest eigenvalue for two groups that behave differently prior to the seizure. The pre-seizure increase of the smallest eigenvalue is preserved in the calculation for the subgroup Fp1, Fp2, F4, F8, C4, Fz, i.e. electrodes positioned at frontopolar, frontal right and central right locations of the scalp (Fig. 5a). The duration of this increase is comparable to the duration in the full data set. The fluctuations of the eigenvalue are larger than those of the calculation with the full data set, which is due to the smaller number of time series considered. Fig. 5b shows that no pre-seizure increase of the smallest eigenvalue is found for a group of parietal, temporal and occipital electrodes (P3, P4, T5, T6, Cz, Pz). Note, however, that this group marks the duration as well as the offset of the seizure clearer than the group chosen for Fig. 5a. A third group of time series from electrodes (F3, C3, C4, O1, O2, T3) does not show any preseizure increase and behaves qualitatively as the second group in Fig. 5b. A change of one of the members in the first group in Fig. 5a leads to a smaller pre-seizure increase of the smallest eigenvalue, whereas a change of one member in the second and third group does not lead to a qualitative change in the temporal evolution of the smallest eigenvalue. We thus confirm the finding of a cluster structure within the set of time series.

\section{Discussion}

The eigenvalues of the time-dependent correlation matrix can be used to efficiently analyze temporal changes of crosscorrelations in multivariate data with a small number of time series. We demonstrated how the correlation matrix formalism introduced in [5] is applied to characterize the transition to epileptic seizures in human EEG. The complex, apparently random correlation pattern of ongoing electric activity leads to a comparatively stable distribution of eigenvalues in seizure-free segments of the EEG. However, some non-randomness of the dynamics is reflected in the separation of the largest eigenvalue from its nearest neighbor [2,3] but also in the separation of the smallest eigenvalue [5]. This distribution shows random fluctuations and is affected by measurement artifacts but does not show any clear long-term trend as the time series approaches the seizure (Figs. 1b and 1c). This is consistent with the common understanding of the present type of seizure activity as arising suddenly at all (or most) sites on the scalp from a normal background activity.

During the seizure the eigenvalues of $\mathbf{C}$ undergo a redistribution. The temporal changes of the spectrum of eigenvalues reflect the strength and structure of correlations in the data set. Global changes lead to the separation of one or more of the largest eigenvalues with simultaneous decrease of some or all of the smaller ones. In accordance with the standard interpretation that eigenvalues separated from the bulk represent genuine information [2,3], the increased (decreased) level of the largest (smallest) eigenvalue(s) during the seizure reflects a more correlated state of electric activity when compared to seizure-free periods. In our case, the separation of the two largest eigenvalues from their neighbors (as shown in Fig. 1b) indicates a strong deterministic component in the time series. According to the study in [5] this is consistent with the presence of two correlated groups of time series that are mutually uncorrelated. This is confirmed by the nearest neighbor distributions of the distances between the few largest eigenvalues (Fig. 2). The fact that this increase is compensated by a decrease in most other eigenvalues (Fig. 1c) together with the finding that most components of the eigenvector corresponding to the largest eigenvalue show a preferred orientation (Fig. 3), implies that virtually all electrodes contribute to the well-pronounced correlation pattern during the seizure. This is consistent with the well-known fact that in this type of epilepsy the seizure activity is picked up from all scalp electrodes. Qualitatively similar behavior of the spectrum of eigenvalues in this respect is found for all other recordings where typical absence seizures are encountered.

While previous application of random matrix theory to time series analysis focused almost exclusively on the statistical properties of the "bulk" and concluded that only well-separated largest eigenvalues contain genuine information about the nonrandom correlation structure, we have demonstrated that the inspection of the smallest eigenvalues allows to extract additional details, in particular about changes of correlations between subsets of time series. As was shown with numerical examples in [5], the complete analysis of the smaller eigenvalues in principle allows a hierarchical structuring of the extent of correlations, from local to global. The importance of this finding lies in the fact that if a correlation change is comparatively weak, it may not provoke significant changes in the largest eigenvalues. One takes advantage of the fact that a change of an eigenvalue at the upper end of the spectrum is accompanied by an equivalent counter-change on the other end such that the trace of $\mathbf{C}$ stays constant in time. Given the smaller absolute value of the smallest eigenvalues, this leads to a more significant effect on the lower end of the spectrum (a larger relative change).

A striking example of this was observed during the transition to the seizure. Using relative eigenvalues we were able to detect local changes of correlations in periods of a few seconds before the onset of seizure activity (Fig. 4d). In all cases investigated the smallest relative eigenvalue was found to rise drastically (Table 1). This can be interpreted as a subtle loss of correlations confined to only a few time series during that period.

The changes of the eigenvalues as such do not permit to distinguish between a loss of (positive) correlations and a loss of (negative) anti-correlations. Both would lead to a decreased separation of the eigenvalues at the extremes of the spectrum from the bulk. Nevertheless, taken together with other established features like the increase in autocorrelation after the onset of a seizure, this localized pre-seizure loss of crosscorrelations (as indicated by the smallest eigenvalue of $\mathbf{C}$ ) might lead to an improved characterization of the transition to generalized epileptic seizures. In particular, the demonstrated application of C-matrix and RMT measures allows a characterization of time-dependent correlation patterns in experimental time series.

For patient 5 we were able to show explicitly that the preseizure loss of correlation is confined to a subgroup of elec- 
trodes on the scalp (Fig. 5). This allows a clear characterization of the sites that are possibly participating (Fig. 5a) and those that are not part of the temporary correlation loss (the electrodes chosen for Fig. 5b and the remaining ones). Such a differentiation is consistent with topographic findings on the onset of absence seizures [10] and might be of clinical relevance. In our particular case, we are able to conclude that the pre-ictal correlation change is restricted to the time series in the first group.

Using the results with artificial data from [5] we can thus outline a model for the correlation changes as it is suggested by the studied cases of generalized absence seizures. The background activity in the awake resting state shows a relatively stable correlation pattern that seems to be typical of a patient. In an interval of a few seconds before the visible occurrence of spike-wave complexes in the EEG our method allows the detection of significant changes that can be described by a correlation loss which appears to come from a small group of electrodes. Such a conclusion can be drawn only from the time-resolved application of the $\mathrm{C}$-matrix formalism and the way in which the RMT measures are calculated individually for each of the distances [9]. To our knowledge, this conclusion is of importance because the onset of generalized seizures is generally accepted to be spontaneous and a characteristic correlation pattern prior to the seizure has not been reported before. As soon as the spike-wave complexes set in, there is a strong overall increase in cross-correlations which is maintained during the seizure period. We found that the increase is provoked by two essentially uncorrelated groups of electrodes (patients 1-4) or only one group of electrodes (patient 5). After the seizure period, the typical correlation pattern of the resting activity is resumed.

To summarize, the presented tools allow the efficient extraction of information about temporal and spatial correlation changes from multivariate data. As no further knowledge about the underlying system is required, the method should be widely applicable. In particular, we suggest it for the investigation of situations where a detailed understanding of the mechanisms leading to global correlation changes is of interest, like seismic data with earthquakes (see e.g. [11]), and stock market data with "crashes" (cf. [12]). As the method is not very time-consuming, a real-time implementation might be tried.

\section{Acknowledgements}

Work was supported by CONACyT Mexico (project 40885F). We thank Andreas Galka for preparation of the data, and Yurytzy López and Christian Rummel for fruitful discussion. G.B. gratefully acknowledges support from the Klaus Tschira Foundation.

\section{References}

[1] R.J. Muirhead, Aspects of Multivariate Statistical Theory, J. Wiley \& Sons, New York, 1982.

[2] V. Plerou, P. Gopikrishnan, B. Rosenow, L.A. Nunes, H.E. Stanley, Phys. Rev. Lett. 83 (1999) 1471;

V. Plerou, P. Gopikrishnan, B. Rosenow, L.A. Nunes, T. Guhr, H.E. Stanley, Phys. Rev. E 65 (2002) 066126.

[3] L. Lalaux, P. Cizeau, J.-P. Bouchaud, M. Potters, Phys. Rev. Lett. 83 (1999) 1467.

[4] J. Wishart, Biometrika A 20 (1928) 32.

[5] M. Müller, G. Baier, A. Galka, U. Stephani, H. Muhle, Phys. Rev. E 71 (2005) 046116.

[6] Z. Rogowski, I. Gath, E. Bental, Biol. Cybern. 42 (1981) 9.

[7] B. Hjorth, Amer. J. EEG Techn. 20 (1980) 121.

[8] M.L. Mehta, Random Matrices, Elsevier, Amsterdam, 2004; T. Guhr, A. Müller-Groeling, H.A. Weidenmüller, Phys. Rep. 299 (2000) 189

[9] M. Müller, Y. López, C. Rummel, G. Baier, A. Galka, U. Stephani, H. Muhle, Phys. Rev. E 74 (2006) 041119.

[10] E. Rodin, in: E. Niedermeyer, F. Lopes da Silva (Eds.), Electroencephalography, fourth ed., Lippincott Williams \& Wilkins, Philadelphia, 1999, p. 1191.

[11] W.L. Ellsworth, G.C. Beroza, Science 268 (1995) 851.

[12] D. Sornette, W.-X. Zhou, Int. J. Forecasting 22 (2006) 153. 